\title{
A Short-Cut Method for Computing Positive Variance Component Estimates
}

\author{
JOACHIM HARTUNG \\ Department of Statistics, SFB 475 \\ University of Dortmund \\ Germany
}

\section{Summary}

In a general variance component model with positive variance components a short-cut method is presented that yields almost everywhere for these components positive estimators that are invariant with respect to mean value translation and stay near the unbiasedness.

Key Words: Variance components, Minque, positive Minque, Invariance, approximate unbiased positive variance estimates.

\section{Zusammenfassung}

In einem allgemeinen Varianz-Komponenten-Modell mit positiven Varianzkomponenten wird eine verkürzte Methode vorgestellt, welche für diese Komponenten fast überall positive Schätzer ergibt, die invariant bzgl. Mittelwerttranslationen sind und nahe der Unverzerrtheit bleiben. 


\section{Introduction}

In a general variance components model there is the problem that unbiased quadratic estimators, or also maximum likelihood estimators if a distributional assumption is made, of the variance components can take on with a positive probability negative values for nonnegative variance components. These estimators are put then in such cases equal to zero, which for a usually strictly positive variance component is an unsatisfactory procedure. Therefore in the following a short-cut procedure is derived that overcomes this deficiency by yielding almost everywhere positive variance component estimators staying near the unbiasedness.

\section{The Method}

Let us consider the linear variance component model

$$
z \sim\left(X \beta, \sum_{i=1}^{m} \alpha_{i} \cdot U_{i}\right),
$$

that consists of an $n$-dimensional random variable $z$ with mean value

$$
\mathrm{E} z=X \beta
$$

and variance-covariance matrix

$$
\operatorname{Cov}(z)=\sum_{i=1}^{m} \alpha_{i} \cdot U_{i},
$$

where the $(n \times k)$-design-matrix $X$ and the $m$ symmetric positive semi definite $(n \times n)$-matrices $U_{i}, i=1, \ldots, m$, are known, while the parameter $\beta$ varies in $\mathbb{R}^{k}$ and the parameter $\alpha=$ $\left(\alpha_{1}, \ldots, \alpha_{m}\right)^{T}$ in $\mathbb{R}_{+}^{m}$, the positive orthant of $\mathbb{R}^{m}$, and we assume $\operatorname{rank}(X)<n$, and $\operatorname{Cov}(\Rightarrow)$ to be positive definite.

The problem considered here is to find quadratic estimates for the variance components $\alpha_{1}, \ldots, \alpha_{m}$, which should be positive almost everywhere, i.e. with probability one, and invariant with respect to the group $\Gamma$ of mean value translations,

$$
\Gamma=\left\{z \longmapsto z+X \beta \mid \beta \in \mathbb{R}^{k}\right\}
$$

A maximal invariant linear statistic $y$ with respect to $\Gamma$ is given by

$$
\begin{aligned}
y & =\left(I-X X^{+}\right) z \\
& =\operatorname{Proj}_{\mid \operatorname{Range}(X)^{\perp}} z,
\end{aligned}
$$


where $I$ is the $(n \times n)$-identity-matrix and $X^{+}$is the pseudoinverse of $X$.

We get the reduced (by invariance) linear model

$$
y \sim\left(0, \sum_{i=1}^{m} \alpha_{i} \cdot V_{i}\right), \quad \alpha \in \mathbb{R}_{+}^{m},
$$

where $V_{i}=\left(I-X X^{+}\right) U_{i}\left(I-X X^{+}\right), \mathrm{i}=1, \ldots, \mathrm{m}$.

Let now $A$ be a symmetric $n \times n$-matrix, then a quadratic (invariant) estimator for a linear form $p^{T} \alpha>0, p=\left(p, \ldots, p_{m}\right)^{T}, p \geq 0, p \in \mathbb{R}^{m}$, is given by $y^{T} A y$, and denoting "tr" the trace, its bias is given by

$$
\begin{aligned}
\mathrm{E} y^{T} A y-p^{T} \alpha & =\operatorname{E} \operatorname{tr} A y y^{T}-p^{T} \alpha \\
& =\operatorname{tr} A \mathrm{E}\left(y y^{T}\right)-p^{T} \alpha \\
& =\operatorname{tr} A\left(\sum_{i=1}^{m} \alpha_{i} V_{i}\right)-p^{T} \alpha \\
& =\sum_{i=1}^{m} \alpha_{i}\left(\operatorname{tr} A V_{i}-p_{i}\right),
\end{aligned}
$$

such that $y^{T} A y$ is an unbiased estimator of $p^{T} \alpha$ if

$$
\operatorname{tr} A V_{i}=p_{i}, \quad \text { for all } i=1, \ldots, m,
$$

and a solution $A_{0}$ of these equations, respectively the corresponding quadratic estimation function

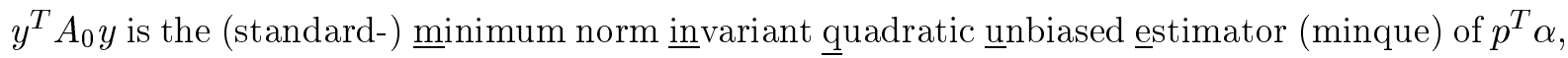
if $A_{0}$ has the minimum norm among all solutions.

Denote Sym the Hilbert space of all symmetric $(n \times n)$-matrices with the inner product of two matrices $A, B \in$ Sym defined by $\operatorname{tr} A B$, which then induces the standard norm $\|A\|=\sqrt{\operatorname{tr} A^{2}}$. Furthermore let PSD denote the cone of positive semi definite matrices in Sym.

If the matrices $V_{1}, \ldots, V_{m}$ are linearly independent, which for simplicity may be assumed here, then the minque $A_{0}$ exists for all $p \in \mathbb{R}^{m}$. Since we only claim $A_{0} \in \mathrm{Sym}$, of course we usually get

$$
y^{T} A y<0 \text { with positive probability }
$$

and only in rare cases $A_{0} \in \mathrm{PSD}$.

Resticting in advance $A$ to be in PSD has the consequence that the equations for unbiasedness are seldom fulfilled, so that these conditions had to be weakened, cf. Seely(1971), Rao(1972), Pukelsheim(1981), Lehmann and Casella(1998), and Hartung(1981), where in section 4 there is also a solution algorithm given, which however needs some numerical effort. 
Therefore in the following a short-cut method is presented that yields an approximation in PSD to $A_{0}$ with a correction for bias.

Let us introduce the linear operator

$$
\begin{aligned}
& \mathrm{Sym} \longrightarrow \mathbb{R}^{m} \\
& \text { g: } A \longmapsto g A=\left(\begin{array}{c}
\operatorname{tr} A V_{1} \\
\vdots \\
\operatorname{tr} A V_{m}
\end{array}\right)
\end{aligned}
$$

then its adjoint $g^{*}$ is given by

$$
\begin{array}{ll}
g^{*}: & \mathbb{R}^{m} \longrightarrow \text { Sym } \\
& a \longmapsto g^{*} a=\sum_{i=1}^{m} \alpha_{i} V_{i}, \quad a=\left(a_{1}, \ldots a_{m}\right)^{T},
\end{array}
$$

such that $g g^{*}$ becomes the Gram-matrix $G$,

$$
g g^{*}=G=\left\{\operatorname{tr} V_{i} V_{j}\right\}_{\substack{i=1, \ldots, m \\ j=1, \ldots, m}}
$$

of which the inverse $G^{-1}$ exists because of the assumed linear independence of $V_{1}, \ldots, V_{m}$.

Denote $g^{+}$the pseudoinverse operator of $g$, then the minque $A_{0}$ is given by

$$
A_{0}=g^{+} p
$$

which because of $g^{+}=g^{*}\left(g g^{*}\right)^{+}$permits the computational representation

$$
A_{0}=\sum_{i=1}^{m} a_{i} \cdot V_{i}, \text { with } a=\left(a_{1}, \ldots, a_{m}\right)^{T}=G^{-1} p .
$$

Let us define now the vectors $b=\left(b, \ldots, b_{m}\right)^{T}$ and $c=\left(q, \ldots, c_{m}\right)^{T}$ by

$$
b_{i}=\left\{\begin{array}{ll}
a_{i}, & \text { if } a_{i}>0 \\
0, & \text { if } a_{i} \leq 0
\end{array}, \quad \text { and } \quad c_{i}= \begin{cases}-a_{i}, & \text { if } a_{i}<0 \\
0, & \text { if } a_{i} \geq 0\end{cases}\right.
$$

then

$$
\begin{aligned}
A_{0} & =g^{*} a \\
& =g^{*} b-g^{*} c \\
& =: A_{1}-A_{2},
\end{aligned}
$$

where $A_{1} \in \mathrm{PSD}, A_{2} \in \mathrm{PSD}$, and

$$
g A_{0}=p, \quad g A_{1}=G b=: q, \quad g A_{2}=G c=: r
$$


such that

$$
p^{T} \alpha=q^{T} \alpha-r^{T} \alpha
$$

with the estimators

$$
\begin{aligned}
\widehat{p^{T} \alpha} & =\widehat{q^{T} \alpha}-\widehat{r^{T} \alpha} \\
& =y^{T} A_{1} y-y^{T} A_{2} y .
\end{aligned}
$$

We assume $A_{2} \neq 0$, otherwise $A_{0} \in$ PSD.

Now $A_{1}$ is an approximation in PSD to $A_{0}$, with the estimate

$$
\begin{aligned}
y^{T} A_{1} y=\widehat{q^{T} \alpha} & =\widehat{p^{T} \alpha}+\widehat{r^{T} \alpha} \\
& <\widehat{r^{T} \alpha}, \text { with positive probability }
\end{aligned}
$$

although

$$
q^{T} \alpha=p^{T} \alpha+r^{T} \alpha>r^{T} \alpha .
$$

An additive correction of $A_{1}$, however, would lead again to possibly negative estimates. So the idea is now to work with a multiplicative correction term $\psi$, such that $\psi \cdot A_{1}$ replaces $A_{0}$ in $\widehat{p^{T} \alpha}$, and $\psi \cdot A_{2}$ replaces $A_{2}$ in $\widehat{r^{T} \alpha}$.

As determination equation for $\psi$ we thus get

$$
E\left\{\psi \cdot \widehat{q^{T} \alpha}+\psi \cdot \widehat{r^{T} \alpha}\right\} \stackrel{!}{=} q^{T} \alpha,
$$

yielding

$$
\psi=\frac{q^{T} \alpha}{q^{T} \alpha+r^{T} \alpha},
$$

and so we define our approximate solution in PSD as

$$
A_{\mathrm{PSD}, \text { appr. }(\psi)}:=\psi \cdot A_{1},
$$

where $\psi$ can be estimated by

$$
\widehat{\psi}=\frac{y^{T} A_{1} y}{y^{T} A_{1} y+y^{T} A_{2} y},
$$

which gives for $p^{T} \alpha$ the desired, approximate estimate

$$
\left(\widehat{p^{T} \alpha}\right)_{\mathrm{PSD}, \operatorname{appr} .(\widehat{\psi})}=\widehat{\psi} \cdot y^{T} A_{1} y \text {. }
$$


We can remark that first simulation results show a good performance of this estimator.

Instead of our easily obtainable decomposition of $A_{0}$, we may also use the spectral decomposition of $A_{0}$ into $A_{1^{*}}-A_{2^{*}}$ as follows:

$$
\begin{aligned}
& A_{1^{*}}:=\sum_{\lambda \in \sigma\left(A_{0}\right)} \max \{0, \lambda\} \cdot P_{\lambda}, \\
& A_{2^{*}}:=A_{1^{*}}-A_{0}
\end{aligned}
$$

where $\sigma\left(A_{0}\right)$ is the spectrum of $A_{0}$ and $P_{\lambda}$ is the projection onto the eigenspace associated with $\lambda$, which needs a higher computational effort.

In more specified models also quite different and more detailed approximations may be derived, cf. e.g. Hartung(1999).

\section{References}

HaRTUnG, J. (1981). Nonnegative minimum biased invariant estimation in variance component models. The Annals of Statistics 9, 278-292.

HaRTung, J. (1999). Ordungserhaltende positive Varianzschätzer bei gepaarten Messungen ohne Wiederholungen. Allgemeines Statistisches Archiv 83, erscheint.

Lehmann, E.L. And Casella, G. (1998). Theory of Point Estimation. 2nd. ed., Springer, New York

Pukelsheim, F. (1981). On the existence of unbiased nonnegative estimates of variance covariance components. The Annals of Statistics 9, 293-299.

RAO, C.R. (1972). Estimation of Variance and Covariance Components in Linear Models. Journal of the American Statistical Association 67, 112-115.

SEelY, J. (1971). Quadratic subspaces and completeness. The Annals of Mathematical Statistics 42, 710-721.

Prof. Dr. Joachim Hartung

Lehrstuhl Statistik mit Anwendungen in den Ingenieurwissenschaften

Fachbereich Statistik der Universität Dortmund

Vogelpothsweg 87

D-44221 Dortmund

Germany 